\title{
Electromagnetic Inversion with Local Power Conservation for Metasurface Design
}

\author{
Trevor Brown, Yousef Vahabzadeh, Christophe Caloz, and Puyan Mojabi
}

\begin{abstract}
A method based on electromagnetic inversion is extended to facilitate the design of passive, lossless, and reciprocal metasurfaces. More specifically, the inversion step is modified to ensure that the field transformation satisfies local power conservation, using available knowledge of the incident field. This paper formulates a novel cost functional to apply this additional constraint, and describes the optimization procedure used to find a solution that satisfies both the user-defined field specifications and local power conservation. Lastly, the method is demonstrated with a two-dimensional (2D) example.
\end{abstract}

Index Terms-Electromagnetic metasurfaces, inverse problems, inverse source problems, optimization, antenna design.

\section{INTRODUCTION}

Metasurfaces have emerged as useful devices for systematically controlling electromagnetic fields [1]-[6]. These subwavelengthly thin structures can perform arbitrary field transformations by imposing appropriate generalized boundary conditions, providing a level of control over some desired field produced by a known incident field. This fundamental ability has led to a variety of applications, including generalized refraction and reflection [7], polarization manipulation [8], [9], spatial processing [10], impedance matching [11], radiation pattern tailoring [12], and others.

In order to design a metasurface to perform a given field transformation, the tangential electric and magnetic fields must be known on either side of the boundary imposed by the metasurface. Most existing design procedures are limited to problems in which the output field is known analytically on the output side of the metasurface. However, this is satisfactory only for well-defined problems such as plane wave refraction [13]. In order to generalize this to incompletely defined problems, we recently developed a design method which allows for more flexible output field specifications [14]. Using this method, referred to as electromagnetic inversion for metasurface design, the field can be specified at arbitrary locations external to the metasurface, either with or without phase (amplitude-only) information. Furthermore, the desired field can also be specified as a set of performance criteria, such as main beam direction(s), null location(s), beamwidth, or polarization. While this method allows for more general field specifications, it does not take advantage of prior knowledge of the incident field and typically requires loss and/or gain to perform the specified field transformation.

In this work, we extend the electromagnetic inversion algorithm of [14] to allow for the design of lossless, passive, and reciprocal metasurfaces. This method uses electromagnetic inversion to solve for a set of tangential output (transmitted) fields that produce some user-specified field, but modifies the inversion process by incorporating an additional step that penalizes solutions that do not satisfy local power conservation (LPC). Note that LPC dictates that for reflectionless metasurfaces the power entering a given unit cell is equal to the power exiting that unit cell in the direction normal to the metasurface. Once an appropriate solution is found that satisfies both the field specifications and LPC, surface susceptibilities (or, other types of parameters such as surface impedances) can be computed to perform the required transformation.

Enforcing LPC necessarily limits the ability to tailor the amplitude of the wavefronts. This can be understood by noting that LPC is concerned with power at the unit cell level, thus, immediately placing a constraint on the amplitude of the wave at the output port of each unit cell. This can be an issue for complex design problems such as antenna pattern synthesis as they often need full control over both amplitude and phase. Recently, different methods have been suggested to enable more amplitude control by considering total power conservation (TPC); e.g., see [15] for different examples. One of these methods [12], [16] uses two metasurfaces instead of one, each of which individually satisfies LPC. However, collectively, the two-metasurface system only satisfies TPC. Herein, we restrict our attention to single metasurfaces and do not consider cascaded structures.

We begin by briefly reviewing the electromagnetic inversion design procedure without LPC. We then discuss and derive the constraint used to enforce LPC, and then describe how the inversion process is modified to account for this new constraint. An example is then presented to demonstrate this idea. Finally, some conclusions and a discussion of possible extensions to this work are presented.

\section{INVERSE SOURCE DESIGN FrAMEWORK}

Herein, we present a brief review of the design method presented in [14], where the main goal is to find tangential fields on the output side of the metasurface that satisfy some set of user-defined field specifications $S$ in some external region of interest (ROI). An overview of the problem is depicted in Figure 1. We denote the input and output surface boundaries of the metasurface as $\Sigma^{-}$and $\Sigma^{+}$, respectively. The tangential fields (denoted as such by the subscript $t$ ) that we require to design the metasurface consist of the total fields on $\Sigma^{-}, \vec{E}_{t}^{-}$and $\vec{H}_{t}^{-}$(consisting of the incident and reflected fields), and the transmitted fields on $\Sigma^{+}, \vec{E}_{t}^{+}$and $\vec{H}_{t}^{+}$. The user-defined specifications $S$ in the ROI fall into three general categories, ordered from most to least specific (i.e., most to least information): 


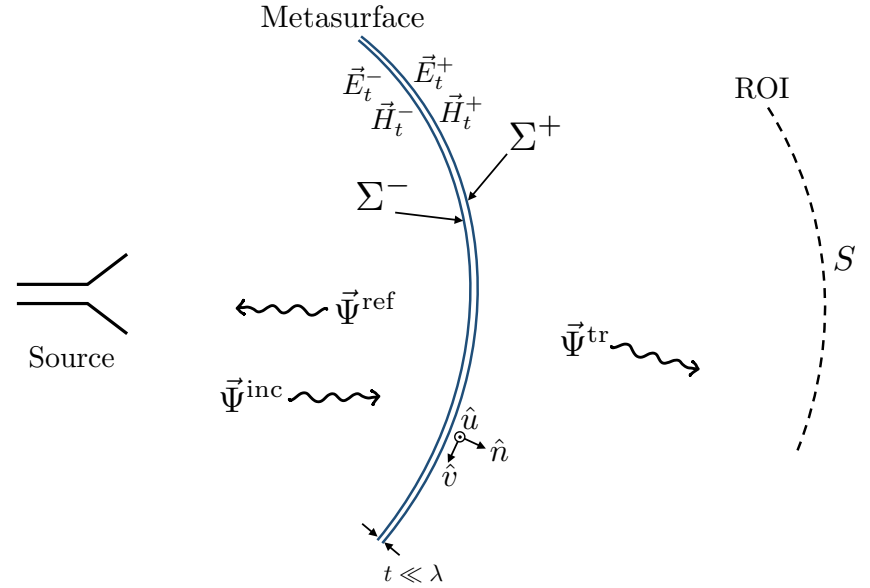

Fig. 1. Overview of the metasurface design problem. The input and output surface boundaries of the metasurface are denoted by $\Sigma^{-}$and $\Sigma^{+}$, respectively. Some source generates an incident field $\vec{\Psi}^{\text {inc }}$ which interacts with the metasurface, producing both a reflected field $\vec{\Psi}^{\text {ref }}$ and a transmitted field $\vec{\Psi}^{\text {tr }}$. The tangential components of the electric and magnetic fields on $\Sigma^{-}$are denoted as $\vec{E}_{t}^{-}$and $\vec{H}_{t}^{-}$, while the tangential fields on $\Sigma^{+}$are denoted as $\vec{E}_{t}^{+}$and $\vec{H}_{t}^{+}$. The user-defined field specifications $S$ are defined on some of interest (ROI) external to the metasurface. Since the metasurface may be of arbitrary shape, we define the local coordinate system $(\hat{u}, \hat{v}, \hat{n})$ on $\Sigma^{+}$, where $\hat{n}$ is the unit outward normal to $\Sigma^{+}$. () 2019 IEEE. Reprinted, with permission, from [14] with minor modifications.

1) Complex (amplitude and phase) field distributions (either in the near-field or far-field regions),

2) Phaseless field distributions (i.e., amplitude-only, power pattern),

3) Far-field performance criteria (i.e., main beam directions(s), null locations, beamwidth, etc.).

The specifications $S$ in the ROI will be inputted to an electromagnetic inverse source algorithm, which we simply refer to as an inversion algorithm. ${ }^{1}$ This inversion algorithm solves for a set of equivalent electric $(\vec{J})$ and magnetic $(\vec{M})$ currents that produce the field specifications in the ROI. It should be noted that a unique solution for the equivalent currents does not exist due to the inherent ill-posedness of the inverse source problem; however, this non-uniqueness is actually advantageous for design problems as it increases the available degrees of freedom [18], [19]. The domain upon which the equivalent currents are determined, commonly referred to as the 'reconstruction surface', is chosen to coincide with the physical boundary imposed by the metasurface. These currents are found by minimizing a data misfit cost functional, which we denote herein as $\mathcal{C}_{1}(\vec{J}, \vec{M})$, using the conjugate gradient method. (This cost functional is a mapping from complex equivalent currents to a real value.) This functional essentially quantifies the difference between the fields generated by the equivalent currents and the field specifications, with the exact form depending on the category of field specifications listed above. (For more details on this data misfit cost functional, see (12), (13), and (20) in [14].)

If Love's equivalence condition is enforced (i.e., null fields

\footnotetext{
${ }^{1}$ In addition to electromagnetic inverse source algorithms, electromagnetic inverse scattering algorithms have also been used for design applications; e.g., see [17].
}

on the input side of the metasurface), then the resulting equivalent currents are related to the desired transmitted fields as

$$
\vec{H}_{t}^{+}=-\alpha \hat{n} \times \vec{J} \quad \text { and } \quad \vec{E}_{t}^{+}=\alpha \hat{n} \times \vec{M},
$$

where $\alpha$ is a real-valued scaling parameter which does not affect the characteristics of the normalized radiated field, but allows for some flexibility that will be utilized in the next section. Once the desired tangential transmitted fields are known, the generalized sheet transition conditions (GSTCs) [20] can be utilized to determine a set of surface susceptibilities modelling the discontinuity from the (known) incident/reflected field and (desired) transmitted field [6]. Assuming a timedependency of $e^{j \omega t}$ and free space on either side of the metasurface, the relationship can be written as

$$
\begin{aligned}
\left(\begin{array}{c}
-\Delta H_{v} \\
\Delta H_{u}
\end{array}\right)=j \omega \epsilon_{0}\left(\begin{array}{ll}
\chi_{\mathrm{ee}}^{u u} & \chi_{\mathrm{ee}}^{u v} \\
\chi_{\mathrm{ee}}^{v u} & \chi_{\mathrm{ee}}^{v v}
\end{array}\right)\left(\begin{array}{l}
E_{u, \mathrm{av}} \\
E_{v, \mathrm{av}}
\end{array}\right) \\
+j \omega \sqrt{\epsilon_{0} \mu_{0}}\left(\begin{array}{ll}
\chi_{\mathrm{em}}^{u u} & \chi_{\mathrm{em}}^{u v} \\
\chi_{\mathrm{em}}^{v u} & \chi_{\mathrm{em}}^{v v}
\end{array}\right)\left(\begin{array}{l}
H_{u, \mathrm{av}} \\
H_{v, \mathrm{av}}
\end{array}\right) \\
\left(\begin{array}{c}
-\Delta E_{u} \\
\Delta E_{v}
\end{array}\right)=j \omega \mu_{0}\left(\begin{array}{ll}
\chi_{\mathrm{mm}}^{v v} & \chi_{\mathrm{mm}}^{v u} \\
\chi_{\mathrm{mm}}^{u v} & \chi_{\mathrm{mm}}^{u u}
\end{array}\right)\left(\begin{array}{l}
H_{v, \mathrm{av}} \\
H_{u, \mathrm{av}}
\end{array}\right) \\
+j \omega \sqrt{\epsilon_{0} \mu_{0}}\left(\begin{array}{ll}
\chi_{\mathrm{me}}^{v v} & \chi_{\mathrm{me}}^{v u} \\
\chi_{\mathrm{me}}^{u v} & \chi_{\mathrm{me}}^{u u}
\end{array}\right)\left(\begin{array}{l}
E_{v, \mathrm{av}} \\
E_{u, \mathrm{av}}
\end{array}\right),
\end{aligned}
$$

where $\omega$ is the angular frequency of the time harmonic fields, and $\epsilon_{0}$ and $\mu_{0}$ are the permittivity and permeability of free space. ${ }^{2}$ The subscripts and superscripts $u$ and $v$ denote the tangential components of the local coordinate system of each unit cell defined by $\hat{u} \times \hat{v}=\hat{n}$ and $\hat{u} \perp \hat{v}$. The $\chi$ terms represent the electric/magnetic (first subscript) surface susceptibility components in the presence of an electric/magnetic (second subscript) field excitation [21]. The spatial dependencies of the $E, H$, and $\chi$ terms, which span the metasurface geometrical surface, have been dropped for brevity. The difference and average fields are defined for an arbitrary field $\vec{\Psi}$ as

$$
\begin{array}{r}
\Delta \vec{\Psi} \triangleq \vec{\Psi}^{\mathrm{tr}}-\left(\vec{\Psi}^{\mathrm{inc}}+\vec{\Psi}^{\mathrm{ref}}\right) \\
\vec{\Psi}_{\mathrm{av}} \triangleq \frac{\left.\vec{\Psi}^{\mathrm{tr}}\right|_{\Sigma^{+}}+\left(\left.\vec{\Psi}^{\mathrm{inc}}\right|_{\Sigma^{-}}+\left.\vec{\Psi}^{\mathrm{ref}}\right|_{\Sigma^{-}}\right)}{2} .
\end{array}
$$

The final step in the design procedure is solving (2) for the non-zero susceptibility terms (depending on the problem, some $\chi$ terms may be assumed to be zero). Once the susceptibilities are determined, they can be implemented in different ways, e.g., as three-layered (dogbone) impedance sheets [22], [23], as will be considered later. Note that in the three-layer impedance sheet approach, it is often assumed that the impedance sheets are purely reactive, and that they are printed on (nearly) lossless dielectric substrates. Therefore, LPC needs to hold when considering this unit cell design approach.

\footnotetext{
${ }^{2}$ The formulation shown here assumes that the normal components of the polarization densities are zero for mathematical simplicity.
} 


\section{ENFORCING LOCAL POWER CONSERVATION (LPC)}

The main limitation of the electromagnetic inversion design procedure presented in [14] is that the synthesized susceptibilities may require (undesirable) loss and/or gain. To overcome this issue, we first require that the input and output fields must satisfy LPC [23], [24]. That is, the real power incident on each unit cell must be equal to the real power transmitted from each unit cell, as enforced by the following equation that must hold along the metasurface, i.e., at each unit cell:

$$
\frac{1}{2} \operatorname{Re}\left(\vec{E}_{t}^{-} \times \vec{H}_{t}^{-*}\right)=\frac{1}{2} \operatorname{Re}\left(\vec{E}_{t}^{+} \times \vec{H}_{t}^{+*}\right)
$$

where 'Re' denotes the real-part operator and the superscript '*' denotes the complex conjugate operator. From this point onwards, we will assume $2 \mathrm{D} \mathrm{TE}_{z}$ polarized fields and a $1 \mathrm{D}$ metasurface along the line $x=0$ (i.e., $\hat{u}=\hat{y}, \hat{v}=\hat{z}$, and $\hat{n}=$ $\hat{x}$ ) for simplicity, although the formulation would still hold for arbitrarily-shaped metasurfaces and 3D fields. We denote the left hand side of (5) evaluated at the $i^{\text {th }}$ unit cell as

$$
p_{i}=\left.\frac{1}{2} \operatorname{Re}\left(E_{y}^{-} \times H_{z}^{-*}\right)\right|_{\text {unit cell } i} .
$$

Note that $p_{i}$ for all $i$ values is known since it corresponds to the input power density at each unit cell of the metasurface. (Herein, we assume a reflectionless metasurface; thus, $E_{y}^{-}$ and $H_{z}^{-}$correspond to the incident electromagnetic field.) Using (6) and the relationship between the equivalent currents and the tangential transmitted fields in (1), we can write the LPC constraint in (5) as the following vector equality

$$
\left[\begin{array}{c}
p_{1} \\
\vdots \\
p_{N}
\end{array}\right]=\frac{\alpha^{2}}{2} \operatorname{Re}\left(\left[\begin{array}{c}
\left.M_{z} J_{y}^{*}\right|_{\text {unit cell } 1} \\
\vdots \\
\left.M_{z} J_{y}^{*}\right|_{\text {unit cell N }}
\end{array}\right]\right)
$$

where $N$ is the total number of unit cells. The above vector equality can then be compactly written as

$$
\mathbf{p}=\frac{\alpha^{2}}{2} \operatorname{Re}\left(\mathbf{M} \odot \mathbf{J}^{*}\right)
$$

where $\mathbf{J}$ and $\mathbf{M}$ are discretized complex vectors of the equivalent currents $J_{y}$ and $M_{z}$ at each unit cell; i.e., $\mathbf{J} \in \mathbb{C}^{N}$ and $\mathbf{M} \in \mathbb{C}^{N}$. In addition, ' $\odot$ ' represents the element-wise Hadamard product. The vector $\mathbf{p} \in \mathbb{R}^{N}$ is a discrete vector of the real incident power calculated at each unit cell, with the $i^{\text {th }}$ element of $\mathbf{p}$ equal to $p_{i}$.

Separating the equivalent currents into their real and imaginary parts, denoted by the subscripts 'R' and 'I', (8) becomes

$$
\begin{aligned}
\mathbf{p} & =\frac{\alpha^{2}}{2} \operatorname{Re}\left\{\left(\mathbf{M}_{\mathbf{R}}+j \mathbf{M}_{\mathrm{I}}\right) \odot\left(\mathbf{J}_{\mathrm{R}}-j \mathbf{J}_{\mathrm{I}}\right)\right\} \\
& =\frac{\alpha^{2}}{2}\left(\mathbf{J}_{\mathrm{R}} \odot \mathbf{M}_{\mathrm{R}}+\mathbf{J}_{\mathrm{I}} \odot \mathbf{M}_{\mathrm{I}}\right) .
\end{aligned}
$$

At this point, the restriction imposed by the LPC constraint becomes obvious. In [14], the equivalent currents had four degrees of freedom (i.e. $\mathbf{J}_{R}, \mathbf{J}_{I}, \mathbf{M}_{R}$, and $\mathbf{M}_{\mathrm{I}}$ ) to satisfy the field specifications, but as shown in (9), the LPC constraint reduces the degrees of freedom to three. In other words, enforcing LPC results in a reduction in the dimension of the solution space, and may exclude some solutions that would otherwise satisfy the field constraints in an optimal manner.
We can now formulate a cost functional, say $\mathcal{C}_{2}$, to quantify the LPC constraint and include it in the design procedure. This term is formulated from (9) as

$$
\mathcal{C}_{2}(\mathbf{J}, \mathbf{M})=\frac{\left\|\mathbf{J}_{\mathrm{R}} \odot \mathbf{M}_{\mathrm{R}}+\mathbf{J}_{\mathrm{I}} \odot \mathbf{M}_{\mathrm{I}}-\frac{2}{\alpha^{2}} \cdot \mathbf{p}\right\|_{2}^{2}}{\left\|\frac{2}{\alpha^{2}} \cdot \mathbf{p}\right\|_{2}^{2}}
$$

where $\|\cdot\|_{2}$ represents an $L_{2}$ norm. Since we have complete freedom in selecting the scaling parameter $\alpha$, it should be chosen in a way that minimizes (10) for a given set of currents. Therefore, when (10) is evaluated, the parameter $\alpha$ that results in the minimum of (10) is used.

\section{Methodology}

Our unknowns consist of the separated real and imaginary parts of the electric and magnetic equivalent currents, which we collectively write for convenience as

$$
\mathbf{x}=\left[\mathbf{J}_{R} ; \mathbf{J}_{I} ; \mathbf{M}_{R} ; \mathbf{M}_{I}\right] \in \mathbb{R}^{4 N}
$$

where ';' denotes the column-wise vector concatenation. First, as described in [14], $\mathcal{C}_{1}(\mathbf{x})$, which includes the desired field specifications, is minimized without the LPC constraint using the conjugate gradient method. This provides an estimate for $\mathrm{x}$ that satisfies the field constraints and Love's condition prior to applying the LPC constraint. This estimate of $\mathbf{x}$ is denoted as $\mathbf{x}_{0}$. Next, we minimize

$$
\mathcal{C}(\mathbf{x})=\underbrace{\mathcal{C}_{1}(\mathbf{x})}_{\text {specifications }}+\kappa \underbrace{\mathcal{C}_{2}(\mathbf{x})}_{\text {LPC }},
$$

where $\kappa$ is a real-valued scalar weighting parameter used to balance the contribution of $\mathcal{C}_{2}$ (LPC) with respect to $\mathcal{C}_{1}$ (field specifications). Particle swarm optimization (a global optimization technique) is used to minimize (12), rather than a gradient-based technique, due to the increased nonlinearity introduced by $\mathcal{C}_{2}(\mathbf{x})$. The initial particle states are set to $\mathbf{x}_{0}$ and the absolute search space bounds are set to be $10 \%$ above the maximum absolute value of $\mathbf{x}_{0}$. Once convergence is reached, the required tangential fields are obtained using (1). ${ }^{3}$

Next, the susceptibility components required to support the desired transformation must be computed. As noted in [23], if we want to support a transformation of this nature without using loss and/or gain, we require more degrees of freedom than afforded by only $\overline{\bar{\chi}}_{\mathrm{ee}}$ and $\overline{\bar{\chi}}_{\mathrm{mm}}$ (tensors collectively representing the various $\chi_{\mathrm{ee}}$ and $\chi_{\mathrm{mm}}$ terms in (2), respectively). One way to overcome this limitation is by allowing the bianisotropic terms $\overline{\bar{\chi}}_{\mathrm{em}}$ and $\overline{\bar{\chi}}_{\mathrm{me}}$ to be non-zero, introducing magnetoelectric coupling to the metasurface.

Assuming 2D $\mathrm{TE}_{z}$ fields with a 1D metasurface along $x=0,(2)$ simplifies to

$$
\begin{aligned}
& -\Delta H_{z}=\left(j \omega \epsilon_{0} E_{y, \mathrm{av}}\right) \chi_{\mathrm{ee}}^{y y}+\left(j \omega \sqrt{\mu_{0} \epsilon_{0}} H_{z, \mathrm{av}}\right) \chi_{\mathrm{em}}^{y z} \\
& -\Delta E_{y}=\left(j \omega \mu_{0} H_{z, \mathrm{av}}\right) \chi_{\mathrm{mm}}^{z z}+\left(j \omega \sqrt{\mu_{0} \epsilon_{0}} E_{y, \mathrm{av}}\right) \chi_{\mathrm{me}}^{z y}
\end{aligned}
$$

\footnotetext{
${ }^{3}$ In this framework, the number of discrete points used to enforce the field specifications does not typically increase with problem size. Therefore, for larger problems, the number of 'observation' points (related to the rows of the matrix operator involved in computing $\mathcal{C}_{1}$ ) will become dominated by the 'virtual' points at which Love's equivalence condition (null field) is enforced. With this assumption, the computational complexity of evaluating $\mathcal{C}_{1}$ is $O\left(N^{2}\right)$ and $\mathcal{C}_{2}$ is $O(N)$, where $N$ is the number of unit cells.
} 
In order to avoid loss and gain, we first stipulate that $\chi_{\mathrm{ee}}^{y y}$ and $\chi_{\mathrm{mm}}^{z z}$ must be purely real [23]. Next, we note that since $\chi_{\mathrm{ee}}^{y y} \in \mathbb{R}$ and $\chi_{\mathrm{mm}}^{z z} \in \mathbb{R}$ satisfy the first two conditions for losslessness and passivity, the remaining (third) condition for losslessness and passivity, i.e., $\left(\chi_{\mathrm{em}}^{y z}\right)^{*}=\chi_{\mathrm{me}}^{z y}$, must also hold since the field transformation satisfies LPC. If we enforce $\chi_{\mathrm{em}}^{y z}$ and $\chi_{\mathrm{me}}^{z y}$ to be purely imaginary, then any lossless and passive solution will also satisfy the condition of reciprocity, $\chi_{\mathrm{em}}^{y z}=-\chi_{\mathrm{me}}^{z y}$ [13]. This results in four real unknowns (per unit cell) that must satisfy the two complex equations in (13), which can be directly computed assuming the tangential fields on both sides of the metasurface are known.

\section{RESults}

To illustrate the proposed method, we design a reflectionless 1D metasurface specified to transform an incident $\mathrm{TE}_{z}$ plane wave into a desired power pattern (phaseless field information only) specified in the far-field region. The fields are assumed to propagate in $2 \mathrm{D}$ in the $x y$ plane, with the metasurface placed at $x=0$. The frequency is $10.5 \mathrm{GHz}$ and the metasurface unit cells are $\lambda / 6$ in length, where $\lambda$ represents the free space wavelength. The designed region of the metasurface extends from $y=-2.5 \lambda$ to $y=2.5 \lambda$, with absorbing elements placed along the rest of the $x=0$ line. The incident field is a normally incident uniform plane wave. The desired phaseless power pattern is produced by simulating an array of 9 uniformly spaced elementary dipoles along the $y$-axis between $y=-2 \lambda$ and $y=2 \lambda$. The specified desired far-field power pattern is computed for $-90^{\circ} \leq \varphi \leq 90^{\circ}$ and shown (in red) in Figure 2 .

The surface on which the equivalent currents are reconstructed is chosen to coincide with the metasurface, with the same $\lambda / 6$ discretization. First, a solution is found by minimizing the cost functional without the LPC constraint. This solution is then used to initialize a particle swarm optimization algorithm that minimizes (12) using a swarm size of 200 and a scaling factor of 0.1 for $\kappa$. The far-field pattern corresponding to the equivalent currents from the particle swarm optimization stage is shown in Figure 2 to demonstrate the decrease in pattern accuracy that must be made to enforce the LPC constraint. The resulting solution is then used to compute the four susceptibility terms using (1) and (13), which are necessarily passive, lossless, and reciprocal.

We then simulate the designed metasurface in ANSYS HFSS using the method described in [25]. This technique implements each unit cell using a three-layer admittance sheet topology [26], using the conversion between susceptibilities and admittances explicitly stated in [25]. The unit cells are then modelled in HFSS using three impedance boundary conditions, between which exist two substrate layers. The substrate used in this example is Rogers RO3010 $\left(\epsilon_{r}=10.2, \tan \delta=0.0022\right)$ with each layer having a thickness of 50 mil. Additionally, metallic baffles have been placed between the unit cells, as in [22]. The total electric field resulting from this simulation is shown in Figure 3, and the far-field pattern associated with this simulation is shown in Figure 2. These results show that the main features of the desired power pattern have been generated with only minor reflections, despite some deviation in the

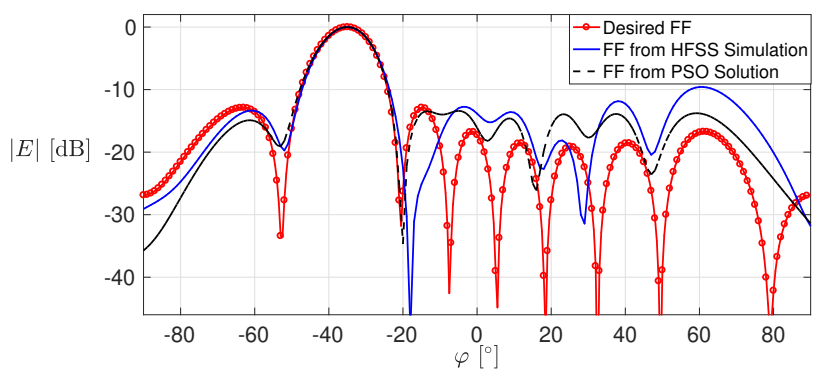

Fig. 2. Far-field power pattern produced by the equivalent currents generated after enforcing LPC using PSO (dashed black curve), the power pattern produced by the HFSS simulation of the designed metasurface (solid blue curve), and specified power pattern (solid red curve with circular markers).

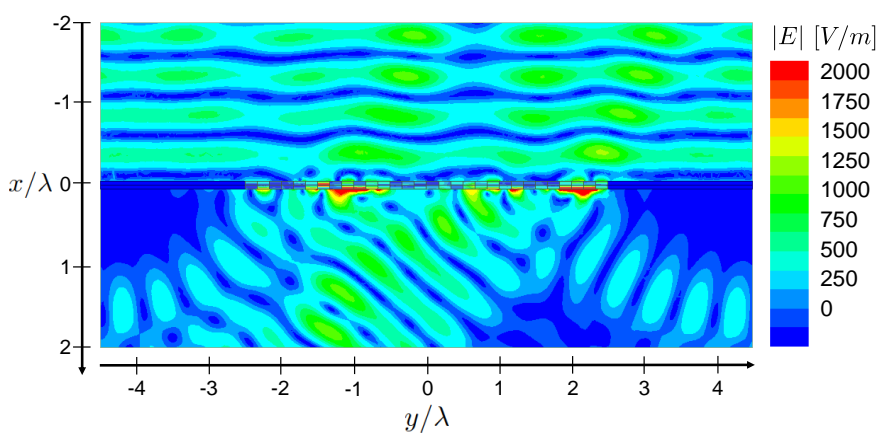

Fig. 3. Electric field amplitude when the designed metasurface is implemented using the three-layer admittance sheet topology and illuminated by a normally incident uniform plane wave, simulated using ANSYS HFSS.

sidelobes. As noted earlier, this might be attributed to the fact that LPC does not allow for full control over the amplitude of the transmitted field. The transmission efficiency, defined as the ratio of the real power transmitted through the metasurface to the real power incident on the metasurface, is $78.7 \%$.

\section{CONCLUSION}

A general metasurface design method was extended to ensure that the resulting field transformation satisfies local power conservation, allowing for the design of passive, lossless, and reciprocal metasurfaces. A constraint on the equivalent currents was derived from the local power conservation relationship, and incorporated into the design procedure using a secondary optimization step. Non-zero magnetoelectric coupling terms are introduced to compensate for the loss of degrees of freedom resulting from excluding loss and gain. A preliminary 2D example was shown for the design of a passive, lossless, and reciprocal metasurface attempting to produce a specified (phaseless) power pattern, with relatively good agreement.

\section{ACKNOWLEDGMENT}

The authors acknowledge the financial support of the Natural Sciences and Engineering Research Council (NSERC) of Canada and the Canada Research Chair (CRC) Program. We would also like to thank the Canadian Microelectronics Corporation (CMC) for the provision of ANSYS HFSS. 


\section{REFERENCES}

[1] C. L. Holloway, E. F. Kuester, J. A. Gordon, J. O'Hara, J. Booth, and D. R. Smith, "An overview of the theory and applications of metasurfaces: The two-dimensional equivalents of metamaterials," IEEE Antennas and Propagation Magazine, vol. 54, no. 2, pp. 10-35, 2012.

[2] C. Pfeiffer and A. Grbic, "Metamaterial Huygens' surfaces: Tailoring wave fronts with reflectionless sheets," Phys. Rev. Lett., vol. 110, p. 197401, May 2013.

[3] M. Selvanayagam and G. Eleftheriades, "Discontinuous electromagnetic fields using orthogonal electric and magnetic currents for wavefront manipulation," Optics Express, pp. 14409-14429, 2013.

[4] S. Tretyakov, "Metasurfaces for general transformations of electromagnetic fields," Philos. Trans. Royal Soc. A: Mathematical, Physical and Engineering Sciences, vol. 373, no. 2049, p. 20140362, 2015.

[5] G. Minatti, M. Faenzi, E. Martini, F. Caminita, P. De Vita, D. GonzálezOvejero, M. Sabbadini, and S. Maci, "Modulated metasurface antennas for space: Synthesis, analysis and realizations," IEEE Transactions on Antennas and Propagation, vol. 63, no. 4, pp. 1288-1300, April 2015.

[6] K. Achouri and C. Caloz, "Design, concepts and applications of electromagnetic metasurfaces," Nanophotonics, vol. 7, no. 6, pp. 1095-1116, 2018.

[7] V. S. Asadchy, M. Albooyeh, S. N. Tcvetkova, A. Díaz-Rubio, Y. Ra'di, and S. Tretyakov, "Perfect control of reflection and refraction using spatially dispersive metasurfaces," Physical Review B, vol. 94, no. 7, p. 075142, 2016.

[8] C. Pfeiffer and A. Grbic, "Bianisotropic metasurfaces for optimal polarization control: Analysis and synthesis," Physical Review Applied, vol. 2, no. 4, p. 044011, 2014.

[9] M. Selvanayagam and G. V. Eleftheriades, "Polarization control using tensor Huygens surfaces," IEEE Transactions on Antennas and Propagation, vol. 62, no. 12, pp. 6155-6168, 2014.

[10] K. Achouri, G. Lavigne, M. A. Salem, and C. Caloz, "Metasurface spatial processor for electromagnetic remote control," IEEE Transactions on Antennas and Propagation, vol. 64, no. 5, pp. 1759-1767, 2016.

[11] A. H. Dorrah, M. Chen, and G. V. Eleftheriades, "Bianisotropic Huygens' metasurface for wideband impedance matching between two dielectric media," IEEE Transactions on Antennas and Propagation, vol. 66, no. 9, pp. 4729-4742, Sep. 2018.

[12] A. H. Dorrah and G. V. Eleftheriades, "Bianisotropic Huygens' metasurface pairs for nonlocal power-conserving wave transformations," IEEE Antennas and Wireless Propagation Letters, vol. 17, no. 10, pp. 17881792 , Oct 2018.

[13] K. Achouri, M. A. Salem, and C. Caloz, "General metasurface synthesis based on susceptibility tensors," IEEE Transactions on Antennas and Propagation, vol. 63, no. 7, pp. 2977-2991, 2015.

[14] T. Brown, C. Narendra, Y. Vahabzadeh, C. Caloz, and P. Mojabi, "On the use of electromagnetic inversion for metasurface design," IEEE Transactions on Antennas and Propagation, vol. 68, no. 3, pp. 18121824, March 2020.

[15] A. H. Dorrah, G. Egorov, and G. V. Eleftheriades, "Non-local power wave transformations using omega bianisotropic Huygens' metasurface pairs," in 2019 URSI International Symposium on Electromagnetic Theory (EMTS), May 2019, pp. 1-4.

[16] V. G. Ataloglou, A. H. Dorrah, and G. V. Eleftheriades, "Design of compact Huygens' metasurface pairs with multiple reflections for arbitrary wave transformations," arXiv:2003.13204, Mar. 2020.

[17] L. Di Donato, T. Isernia, G. Labate, and L. Matekovits, "Towards printable natural dielectric cloaks via inverse scattering techniques," Scientific Reports, vol. 7, no. 1, p. 3680, 2017.

[18] G. Oliveri, E. T. Bekele, M. Salucci, and A. Massa, "Array miniaturization through qcto-si metamaterial radomes," IEEE Transactions on Antennas and Propagation, vol. 63, no. 8, pp. 3465-3476, 2015.

[19] M. Salucci, A. Gelmini, G. Oliveri, N. Anselmi, and A. Massa, "Synthesis of shaped beam reflectarrays with constrained geometry by exploiting nonradiating surface currents," IEEE Transactions on Antennas and Propagation, vol. 66, no. 11, pp. 5805-5817, Nov 2018.

[20] M. M. Idemen, Discontinuities in the electromagnetic field. John Wiley \& Sons (IEEE Press series on electromagnetic wave theory; 40), 2011.

[21] M. Dehmollaian, N. Chamanara, and C. Caloz, "Wave scattering by a cylindrical metasurface cavity of arbitrary cross section: Theory and applications," IEEE Transactions on Antennas and Propagation, vol. 67, no. 6, pp. 4059-4072, 2019.

[22] G. Xu, S. V. Hum, and G. V. Eleftheriades, "Augmented Huygens' metasurfaces employing baffles for precise control of wave transformations," IEEE Transactions on Antennas and Propagation, vol. 67, no. 11, pp. 6935-6946, June 2019.
[23] G. Lavigne, K. Achouri, V. S. Asadchy, S. A. Tretyakov, and C. Caloz, "Susceptibility derivation and experimental demonstration of refracting metasurfaces without spurious diffraction," IEEE Transactions on Antennas and Propagation, vol. 66, no. 3, pp. 1321-1330, 2018.

[24] A. Epstein and G. V. Eleftheriades, "Passive lossless Huygens metasurfaces for conversion of arbitrary source field to directive radiation," IEEE Transactions on Antennas and Propagation, vol. 62, no. 11, pp. 5680-5695, 2014.

[25] T. Brown, Z. Liu, and P. Mojabi, "Full-wave verification of an electromagnetic inversion metasurface design method," TechRxiv preprint, Jan 2020.

[26] J. P. Wong, A. Epstein, and G. V. Eleftheriades, "Reflectionless wideangle refracting metasurfaces," IEEE Antennas and Wireless Propagation Letters, vol. 15, pp. 1293-1296, 2015. 